\title{
Consciousness can overflow report: novel evidence from attribute amnesia of a single stimulus
}

\author{
Rui Wang, Yingtao Fu, Luo Chen, Yutong Chen, Jifan Zhou, Hui Chen \\ Department of Psychology and Behavioral Sciences, \\ Zhejiang University, China
}

Data and methods were posted on the Open Science Framework:

https://osf.io/nce $7 \mathrm{f} /$

Running head: Attribute amnesia favors the overflow of consciousness

Words: 2932

Rui Wang and Yingtao Fu contributed equally to this work

Correspondence concerning this article should be addressed to Hui Chen or Jifan Zhou, Department of Psychology and Behavioral Sciences, Zhejiang University, Xixi Campus, 148 Tianmushan Road, Hangzhou, 310007, China, Emails: chenhui@zju.edu.cn or jifanzhou@zju.edu.cn 


\begin{abstract}
Subjective report has been used as a measurement of consciousness. However, we usually experience seeing much more than what can be reported. This relates to a longstanding controversy regarding the nature of visual consciousness; that is, whether it is confined to cognitive access and can be directly measured by reportability, or whether it is rich and overflows report. Most debate previously concentrated on the unattended information, but acquiesced that information under attentional focus would reach consciousness and be reportable. This study sought to further address the debate from a new perspective, through directly testing whether fully attended supraliminal information is necessarily reportable with a variant of attribute amnesia. Participants were asked to judge the parity of a single number (Experiments $1 \& 3$ ) or whether a Chinese character referred to furniture (Experiments $2 \& 4$ ). After several trials, they were unexpectedly asked to report the target identity. The results consistently showed that participants could not correctly report the target identity, indicating that fully attended information that was consciously perceived could sometimes overflow report. These findings not only provide novel evidence for the overflow argument, but also have crucial implications in the relationship between consciousness and working memory.
\end{abstract}

Keywords: Consciousness, overflow, attribute amnesia, attention and working memory 


\section{Introduction}

How can an experimenter decide whether the participants become conscious of a stimulus? Traditionally, conscious perception has been evaluated by relying on subjective report, typically on a trial-by-trial basis (Dehaene, Changeux, Naccache, Sackur, \& Sergent, 2006; Persuh, 2018; Weiskrantz, 1997). However, this approach has been criticized because both intuition (people often feel that they have seen more than they can report) and some empirical findings (e.g., iconic memory) seem to suggest that we consciously experience a rich visual world, despite only being able to report a few elements at any given moment (Block, 1995; Sperling, 1960). This is related to a longlasting debate regarding the nature of consciousness. Specifically, this debate is about whether visual consciousness is severely limited by cognitive access and thus can be measured by direct reports, or whether the consciousness is rich and overflows report.

According to the no-overflow view, the nature of visual consciousness is quite sparse due to limitations of cognitive processes, such as the focus of attention or working memory capacity (Cohen \& Dennett, 2011; Dehaene et al., 2006; Stazicker, 2011). Two well-known blindness phenomena have supported the sparse consciousness position, namely Change Blindness $(C B)$ and Inattentional Blindness $(I B)$. $C B$ shows a failure to detect obvious changes to a visual scene (e.g., person substitution, Simons \& Levin, 1998), while $I B$ reveals an inability to notice an otherwise prominent stimulus (e.g., a gorilla, Simons \& Chabris, 1999) when attention is occupied elsewhere. These demonstrations of blindness suggest that consciousness is extremely limited by the focus of attention. 
However, the overflow view proposed by Ned Block $(1995,2011)$ argues that despite participants being unable to explicitly report the presence of a change or a stimulus because of inattention as shown in $C B$ and $I B$, they might yet have phenomenally experienced the unreportable information. In other words, there might exist a phenomenal form of consciousness that could happen without attention and go beyond cognitive access/report. This overflow view has also received a great amount of supporting evidence. The most classical example comes from the Sperling-type experiment (Sperling, 1960), in which participants were shown an array of letters (e.g., three rows of four letters) for a brief period, and could only report 3-4 items out of the whole matrix in a free report task, although they often reported having seen all or almost all of the letters. However, when an auditory cue (indicating which row should be reported) was given timely after the offset of stimulus display, they could report almost any item from that cued row, suggesting there must exist a transient but high-capacity conscious phenomenology before cued attention. Another line of evidence comes from studies with the divided attention paradigm, which shows that participants can well perform a periphery task even when their attention was occupied by a central demanding task (e.g., Li, VanRullen, Koch, \& Perona, 2002; Matthews, Schröder, Kaunitz, van Boxtel, \& Tsuchiya, 2018; Reddy, Reddy, \& Koch, 2006; Reddy, Wilken, \& Koch, 2004). This empirical evidence seems to indicate that consciousness can be experienced outside the attentional focus and overflows reportability.

In a summary of evidence either supporting (Sperling task; divided attention task) or against $(C B ; I B)$ overflow view, the main debate concentrated on whether 
information outside the focus of attention could be consciously experienced and reportable. The no-overflow view claimed that information outside attentional focus cannot be consciously perceived or reported (e.g., $C B$ and $I B$ ) while overflow view argues that even the unattended information could sometimes be phenomenally experienced and reportable (e.g., cuing method in Sperling's task). Unlike the abovementioned debate focusing on unattended information, the current study sought to further address the debate from a new perspective, through directly testing whether fully attended supraliminal information is necessarily reportable by adopting a variation of the recently developed paradigm of attribute amnesia (Chen \& Wyble, 2015, 2016; Chen, Yan, et al, 2019; Chen, Yu, et al., 2019). Despite it has been explicitly or implicitly assumed that fully attended information would be consciously perceived and reportable (De Brigard \& Prinz, 2010; Marchetti, 2012), it remains unknown whether this assumption must be true. If we could demonstrate that even clearly visible information under the focus of attention could not be reportable in some circumstances (i.e., attention overflows report), then we would provide stronger evidence for the argument of overflow. This logic leads to our present study.

Attribute amnesia was a recently observed counterintuitive phenomenon by Chen and Wyble (2015) that participants failed to report a specific attribute (e.g., identity of a target letter) under the focus of attention that had just reached conscious awareness. In a typical attribute amnesia experiment, participants are asked to report the location of a target item from a search set of four items (e.g., a target letter among three distractor numbers). After several such trials, participants are unexpectedly asked 
to report the identity of the target item that they have just attended and used for the localization task on a surprise test. Critically, while participants were unable to correctly report the identity in the surprise trial, they could well report it in control trials (following immediately after the surprise trial) once they had an expectation to do so. This striking phenomenon suggests even fully attended information that had reached conscious awareness, cannot guarantee its correct report. However, several characteristics of a classic attribute amnesia paradigm might place potential restrictions on the claim that the unreportable information had reached consciousness. First, in the classic attribute amnesia paradigm, the target item is presented together with three distractors and all stimuli are briefly presented on the screen (typically $<250 \mathrm{~ms}$ ) and masked. Therefore, the unreportable information might be rendered unconsciousness due to the transient presentation time and visual interference from distractors and mask. Second, the original attribute amnesia paradigm was a visual search task wherein participants were required to locate the target letter among distractors. Someone might argue that participants had performed the localization task by categorization without accessing its identity (i.e., the target identity might still did not reach conscious awareness). Specifically, it could be that participants are aware that the stimulus is nonnumeric, they then momentarily determine it to be the target and report its position without identifying it. It is also possible that participants locate the target through excluding all three distractors, in which case the target was also not consciously perceived.

Considering the above issues, here we used a modified attribute amnesia 
paradigm by including a single unmasked stimulus without accompanying any distractors. Furthermore, unlike previous studies presenting stimuli briefly, the stimuli in the current study remained on screen until participants responded to it. With this novel design, the current study tested whether the attribute amnesia effect would still be observed. Such a design could significantly increase our confidence that the attended, yet unreportable information has indeed been consciously perceived (at least momentarily) in the first place, and thus would provide stronger evidence for the view of overflow.

\section{Experiment 1}

Experiment 1 sought to test whether the attribute amnesia effect would persist when presenting participants with a single stimulus that remained at the center of the screen until response.

\section{Method.}

Sample size. We performed a priori power analysis using G*Power 3.1 (Faul, Erdfelder, Lang, \& Buchner, 2007) to estimate the appropriate number of participants. The attribute amnesia effect size $(\varphi)$ was estimated as 0.49 based on the results of Chen and Wyble (2015). The power calculation yielded an estimated minimum of 17 participants to detect such effect with $80 \%$ power (with $\alpha$ set to .05 ). We set the sample size as 20 for each experiment or each group in this study. In this entire study, no participant was allowed to participate in more than one experiment.

$\underline{\text { Participants. Forty Zhejiang University undergraduates completed Experiment }}$ 
1 for course credits or 5 Chinese yuan. Informed consent was obtained for each participant prior to each study in accordance with the institution review board at Zhejiang University. All participants had normal, or corrected-to-normal, vision and had no color vision defects. The participants were pseudo randomly assigned to two groups: 20 to the identity-surprise group and 20 to the color-surprise group. No participants were excluded or replaced in this experiment.

Apparatus. The stimuli were presented on a 15.3-inch computer monitor with screen dimensions of $1,366 \times 768$ pixels. The experiment was programmed using MATLAB with the Psychophysics Toolbox extensions (Brainard, 1997; Pelli, 1997). The viewing distance was at approximate $55 \mathrm{~cm}$. Participants made responses through a computer keyboard.

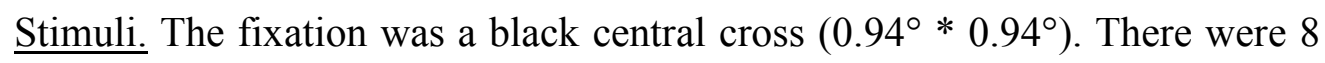
numbers $\left(12,13,14,15,16,17,18,19 ; 1.88^{\circ} * 1.40^{\circ}\right)$, and each of them was displayed in 1 of 4 ink colors: red (RGB: 200, 0, 0), blue (RGB: 0, 0, 200), yellow (RGB: 200, 150, 0), and purple (RGB: 190, 45, 200). All stimuli were displayed on a gray background (RGB: 150, 150, 150) (see Fig. 1).

Procedure and Design. As shown in Fig. 1, each trial started with the central fixation display for a duration that varied between $1000 \mathrm{~ms}$ and $2000 \mathrm{~ms}$. After that, a single colored number, which was randomly selected from a set of eight numbers mentioned above, appeared, and remained at the center of the screen until participants made a response.

Each participant completed 32 trials in this experiment. The first 27 trials (i.e., 
pre-surprise trials) proceeded as just described, in which participants were asked to report the parity of the number by pressing a corresponding key (" $\mathrm{J}$ " for an even number and " $\mathrm{K}$ " for an odd number) as quickly and accurately as possible.

On the $28^{\text {th }}$ trial (i.e., the surprise trial), after $500 \mathrm{~ms}$ of participants' response on the parity task, they were unexpectedly presented with a forced-choice array. For the identity-surprise group, the array included four black numbers: one is the target number that has the same identity with the number presented on that trial and the other three were distractor numbers that have the same parity as the target number (i.e., all four odd numbers or even numbers). The four numbers were presented in a random order, and participants were asked to indicate which one was the target number they had just seen on that trial by pressing one of four corresponding letter keys $(\mathrm{A}, \mathrm{B}, \mathrm{C}$, or D; see Fig. 1). For the color-surprise group, the forced-choice array included four different colored lines (i.e., red, blue, yellow, and purple; see Fig. 1), and participants were asked to indicate which one matched the color of the target number they had just seen on that trial by pressing a corresponding letter key. Following the surprise trial, the participants performed four more control trials of the same format as the surprise trial. 


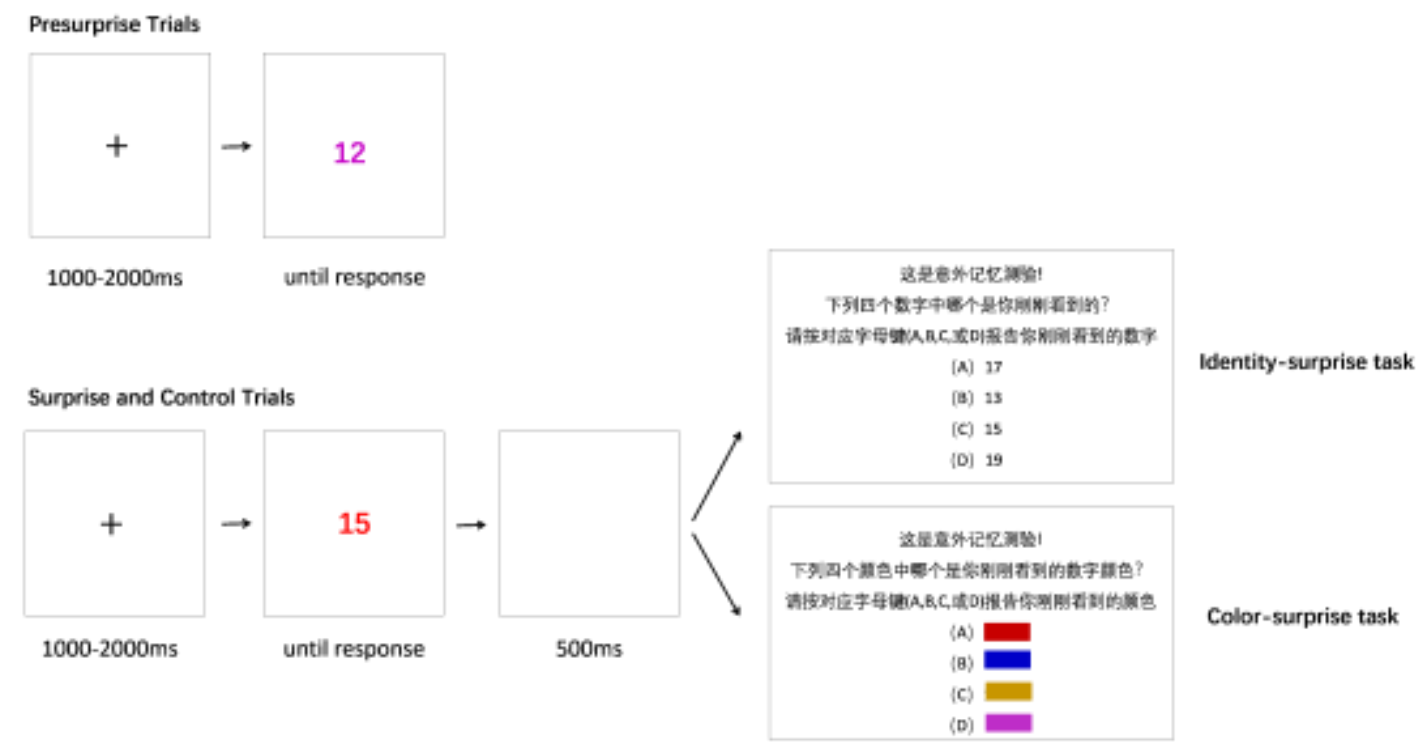

Fig. 1. Sample trial sequence in Experiment 1. The surprise questions were presented in Chinese and the translations were: (1) identity surprise question: "This is a surprise memory test! Which one of following four numbers is the one that you had just seen? Please press a corresponding letter key (A, B, C, or D) to indicate the number"; (2) color surprise question: "This is a surprise memory test! Which one of following four colored lines matches the color of the number that you had just seen? Please press a corresponding letter key (A, B, C, or D) to indicate the colored line."

\section{$\underline{\text { Results and Discussion }}$}

The results of this experiment were depicted in Table 1 . The accuracy of parity report in pre-surprise trials on the identity-surprise and color-surprise groups were $95 \%$ and $96 \%$, respectively, indicating that participants could easily process the number and report its parity with a high accuracy.

Crucially, the attribute amnesia effect was obtained on both color-surprise and identity-surprise groups. For the color-surprise group, only 7 of 20 (35\% correct) participants correctly reported the color of the unitary number on the surprise trial, and 
the performance was largely and significantly improved on the first control trial (i.e., the trial immediately after the surprise trial, $90 \%$ correct $), 35 \%$ vs. $90 \%, \chi^{2}(1, N=40)$ $=10.67^{1}, p<.01, \varphi=.52$. Similarly, for the identity-surprise group, only 11 of $20(55 \%)$ participants were correct in reporting the identity of the number on the surprise trial, which was significantly worse than that on the first control trial, $55 \%$ vs. $95 \%, \chi^{2}(1, N$ $=40)=6.53, p<.05, \varphi=.40$.

The accuracy was consistently high in the following three control trials on both groups: Color-surprise group: 100\%, 100\%, and 100\% correct; Identity-surprise group: $100 \%, 90 \%$, and $100 \%$ correct.

Table 1. The accuracy results of Experiment $1(N=20$ for each group)

\section{Identity-task group}

$\begin{array}{lcccccc} & \text { Pre-surprise } & \text { Surprise } & \text { Control 1 } & \text { Control } 2 & \text { Control } 3 & \text { Control } 4 \\ \text { Parity } & 95 \% & 85 \% & 90 \% & 95 \% & 95 \% & 90 \% \\ \text { Identity } & \text { N/A } & 55 \% & 95 \% & 100 \% & 90 \% & 100 \%\end{array}$

\section{Color-task group}

\begin{tabular}{lcccccc} 
& Pre-surprise & Surprise & Control 1 & Control 2 & Control 3 & Control 4 \\
Parity & $96 \%$ & $95 \%$ & $95 \%$ & $100 \%$ & $95 \%$ & $100 \%$ \\
Color & N/A & $35 \%$ & $90 \%$ & $100 \%$ & $100 \%$ & $100 \%$ \\
\hline
\end{tabular}

Note. $\mathrm{N} / \mathrm{A}=$ Not available

Therefore, Experiment 1 showed that the attribute amnesia effect persisted even

\footnotetext{
${ }^{1}$ Chi-square with Yates corrections was performed if one of the cells is lesser than 5 in all experiments.
} 
under an extreme low load situation wherein there was only a unitary stimulus that remained on the screen until participants made a response. Note that according to previous studies, the parity judgement task cannot be performed without accessing the number meaning (Dehaene, Bossini, \& Giraux, 1993; Fias, Brysbaert, Geypens, \& d'Ydewalle, 1996; Reynvoet \& Brysbaert, 1999). Therefore, this finding provided stronger evidence that the attribute amnesia effect is not caused by a failure of conscious perception, instead, it reflects a report failure of attended information that had just reached conscious awareness.

\section{Experiment 2}

Experiment 2 was designed to test whether the finding of Experiment 1 could be generalized when adopting more complex and meaningful stimuli (i.e., Chinese characters). It could be possible that attribute amnesia would be eliminated or reduced when using complex, meaningful stimuli, as many previous studies showed that participants exhibited an advantage for processing and/or remembering these stimuli (e.g., Brady, Konkle, Alvarez, \& Oliva, 2008; Chen, Yu, et al., 2019; Potter, Nieuwenstein, \& Strohminger, 2008).

\section{Method}

Experiment 2 was identical to Experiment 1 with the following changes. A new group of 20 participants completed this experiment. There were eight Chinese characters in this experiment, four of them correspond to four different types of furniture (i.e., 桌-table, 床-bed, 椅-chair, 灯-lamp) while the other four correspond 
to four different common items encountered in daily life (i.e., 叶-leaf, 桥-bridge, 塔tower, 火-fire). All stimuli were common Chinese characters with a mean word frequency of $60.25(\mathrm{SD}=56.52)$, a mean number of strokes of $8.25(\mathrm{SD}=3.15)$, and a mean familiarity score of $6.69(\mathrm{SD}=0.41)$ according to the Chinese Single-Character Word Database (http://blclab.org/pyscholinguistic-norms-database; Liu, Shu, \& Li, 2007). Each character subtended visual angles of $1.88^{\circ}$ horizontally and $1.40^{\circ}$ vertically. All the stimuli were black which were presented on a gray background (RGB: 150, 150, 150).

On each trial, participants were presented a single Chinese character at the center of the screen and asked to judge whether it was a character for furniture or not as quickly and accurately as possible. Then, in the surprise trial, they were always shown a character for furniture and asked to indicate which one of four possible characters for furniture is the one that they had just seen on that trial. After that, they received four control trials as in Experiment 1.

\section{$\underline{\text { Results and discussion }}$}

As shown in Table 2, the results of Experiment 2 were consistent with Experiment 1, which also showed a clear attribute amnesia effect. Only 10 of $20(50 \%$ correct) participants were correct on the surprise trial, and their performance was dramatically and significantly improved on the first control trial ( $95 \%$ correct), $50 \%$ vs. $95 \%, \chi^{2}(1, N=40)=8.03, p<.01, \varphi=.45$. And the accuracy of the other three control trials was $100 \%$.

This experiment replicated the results of Experiment 1, indicating that attribute 
amnesia could occur for a unitary stimulus presented at the center of the screen, without accompanying any distracting stimuli as in previous attribute amnesia studies. Furthermore, such an effect could not only occur with simple stimuli, but also with the complex and meaningful stimuli (i.e., Chinese characters), further confirming that participants could not always report information that has reached consciousness.

Table 2. The accuracy results of Experiment $2(N=20)$

\begin{tabular}{lclllll}
\hline \multicolumn{2}{r}{ Pre-surprise } & Surprise & Control 1 & Control 2 & Control 3 & Control 4 \\
Type & $96 \%$ & $95 \%$ & $95 \%$ & $100 \%$ & $100 \%$ & $100 \%$ \\
Identity & N/A & $50 \%$ & $95 \%$ & $100 \%$ & $100 \%$ & $100 \%$ \\
\hline
\end{tabular}

Note. N/A = Not available

\section{Experiment 3}

Experiment 3 sought to investigate whether attribute amnesia could still occur when participants encounter a unitary stimulus in a real-world, instead of a laboratory environment as in previous experiments.

\section{Method}

This experiment was similar to Experiment 1 except for the following. A new group of 20 participants completed this experiment. This experiment was run in a realworld context (i.e., in a library room), in which participants were given 32 cards (9 $\mathrm{cm} \times 9 \mathrm{~cm})$ and asked to divide these cards into two parts according to the parity of number presented at the center of each card. They were instructed to finish the task as accurately and quickly as possible. There were 8 possible numbers $(12,13,14,15,16$, 
17, 18, and 19) and each number was displayed in 1 of 4 ink colors (red, blue, yellow, and green), resulting in a total of 32 cards. Once the participants finished the above task, they were unexpectedly presented with a paper including two forced-choice arrays (see Fig.2), and asked to report the identity and color of the number on last card. Noted, unlike previous two experiments, no control trials were conducted in this experiment, since it's so obvious that participants could perform the task perfectly if ask them to do the task again (after the surprise test) in a real-word situation.

\section{$\underline{\text { Results and discussion }}$}

The results of Experiment 3 were displayed in Table 3. The accuracy of the identity and color on surprise trial was $35 \%$ and $40 \%$ respectively, suggesting that the attribute amnesia effect could occur in a real-world situation.

Table 3. The accuracy results of Experiment $3(N=20)$

\begin{tabular}{lll}
\hline & Pre-surprise & Surprise \\
parity & $97 \%$ & N/A \\
identity & N/A & $35 \%$ \\
color & N/A & $40 \%$ \\
\hline
\end{tabular}

Note. $\mathrm{N} / \mathrm{A}=$ Not available

这是意外记忆测验!

下列哪些是您最后看到的数字和颜色?

请选择对应选项
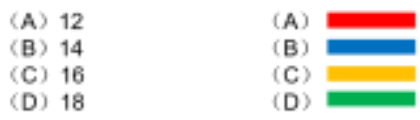

Fig.2. The surprise question of the Experiment 3. The surprise question reads "This is a surprise 
memory test! Please report the corresponding letters to indicate the 'identity' and 'color' of the number on the last card."

\section{Experiment 4}

Note that in Experiments 1 and 2 the stimulus on each trial remained on the screen until participants made a response, which means the presentation time of each stimulus was controlled by participants themselves. Therefore, it might be argued that the increment of performance on the control trials, as compared to the surprise trials, might be due to the fact that participants spent more time in processing the stimuli on control trials wherein they had an expectation to remember the stimuli. Experiment 4 was designed to rule out this possibility by using the same stimulus presentation time for all trials.

\section{Method}

Experiment 4 was identical to Experiment 2 except as follows. An additional 20 participants completed the experiment. After the fixation display for a variable duration $(1,000-2,000 \mathrm{~ms})$, a black Chinese character appeared at the center of the screen for only $150 \mathrm{~ms}$, which was then replaced by a blank screen until participants made a response. Here, participants were also asked to respond as quickly and accurately as possible after the stimulus disappeared.

\section{$\underline{\text { Results and discussion }}$}

As predicted, the attribute amnesia effect persisted (see Table 4). Participants performed poorly $(50 \%$ correct) on the surprise trial, but their performance increased 
on four control trials $(75 \%, 95 \%, 95 \%, 100 \%)$, with the increment reaching significance by the second control trial, $50 \%$ vs. $95 \%, \chi^{2}(1, N=40)=8.03, p<.01, \varphi=.45$. The results showed that attribute amnesia was still obtained when the presentation time of the stimulus was constant on all trials, thus ruling out the above-mentioned possibility that the performance difference between the surprise and control trials was due to the different processing time.

Table 4. The accuracy results of Experiment $4(N=20)$

\begin{tabular}{lllllll}
\hline & Pre-surprise & Surprise & Control 1 & Control 2 & Control 3 & Control 4 \\
Type & $91 \%$ & $100 \%$ & $80 \%$ & $95 \%$ & $95 \%$ & $100 \%$ \\
Identity & N/A & $50 \%$ & $75 \%$ & $95 \%$ & $95 \%$ & $100 \%$ \\
\hline
\end{tabular}

Note. $\mathrm{N} / \mathrm{A}=$ Not available

\section{General Discussion}

The above experiments showed converging evidence that stimuli (e.g., number or character) that was under the focus of attention and consciously perceived could not be reported either in computer-controlled experiments or real-world situations. These results provided stronger support for the overflow argument by demonstrating that even the conscious perception of fully attended information could sometimes overflow report.

\section{Had the attended attribute reached consciousness?}

To support for the overflow view, we should first ensure that the unreported attribute (i.e., identity) had reached consciousness. Kouider and Dehaene (2007) identified two factors for a stimulus to reach consciousness: (i) the input stimulus must 
have enough strength (which can be prevented by masking) and (ii) it must receive topdown attention (which can be prevented by drawing attention to another stimulus or task). In the original attribute amnesia paradigm, where the target was briefly presented and accompanied with distractors and mask, the stimulus input might not have been strong enough and therefore did not satisfy the first criterion. However, both requests are obviously met in the current study. For the first one, the single stimulus remains onscreen without masking until participants make a response, so that the stimulus should have sufficient strength. For the second request, the stimulus does receive top-down attention, since participants must attend to and process its identity to complete the task. What is more, in the absence of sensory competition, as when an object appears in isolation, the cognitive processing load is extremely low. Thus, based on the dominant definition of consciousness, the unreportable information in the current study should have reached consciousness.

Similarly, a phenomenon termed as irrelevance-induced blindness has shown that some participants failed to report one attribute of a single stimulus (Eitam, Shoval, \& Yeshurun, 2015; Eitam, Yeshurun, \& Hassan, 2013). For example, participants were presented with a simple stimulus consisting of two differently colored concentric circles and asked to concentrate on the relevant circle (outer or inner) while ignore the other one. Interestingly, the results showed that participants' performance of reporting the color of the irrelevant circle was significantly worse than the relevant circle in the surprise test, even though the irrelevant and relevant circles could be perceptually integrated into an object. Notably, unlike attribute amnesia, the tested information in 
the irrelevance-induced blindness was totally task-irrelevant and could be selectively ignored according to the studies of feature-selective attention (e.g., Chen, Hoffmann, Albright, \& Thiele, 2012; Jackson, Rich, Williams, \& Woolgar, 2016), and thus did not satisfy the second criterion of consciousness mentioned above. Therefore, we believe that the current study provided stronger evidence that the unreportable information had reached consciousness, compared with irrelevance-induced blindness or previous attribute amnesia studies.

\section{Does consciousness overflow report?}

As we summarized in the introduction, previous overflow supporters focused on the information out of attentional focus, trying to prove that the unattended information could reach consciousness. For attended information, in contrast, there seems a consensus that it could reach consciousness and be reported. This is reasonable, considering that attention has been traditionally seen as a gateway to access consciousness (Dehaene \& Naccache 2001; Lamme, 2010). However, this consensus has been challenged by the current as well as previous attribute amnesia studies, which showed a report failure of well-attended information. We believe that these studies of attribute amnesia provide novel evidence supporting the overflow view. That is, even for the specific information that participants were forced to pay attention and process, they were unable to immediately report it, providing a compelling case that consciousness content overflows report. This expands our understanding of overflow: not only the unattended information, but also attended information could sometimes overflow report. 
The current study implies that the report failure in some blindness phenomena like $C B$ or $I B$ may be due to amnesia, instead of a failure of perception, as even attended information could not be reported. Certainly, this does not mean that there is no perception failure in all cases. Instead, we believe that in some cases, the failure of report is indeed due to a lack of conscious perception, as demonstrated in repeated $I B$ (Ward \& Scholl, 2015). In that study, after participants experienced a typical $I B$ for an unexpectedly red cross moving across the mid-line in a surprise trial, they were explicitly instructed to pay attention to anything unexpected in following trials and immediately report once they saw it. Afterward, there were several trials with the same unexpected red cross moving across the mid-line and participants could successfully report it. On the final critical trial, unlike before, a novel blue letter E moved across the mid-line in the opposite direction, and surprisingly some participants still missed it. This result provides strong evidence that the unexpected stimulus (i.e., blue letter E) is not consciously perceived and thereby leads to a failure of report.

\section{Is reportability always suitable as an index of conscious awareness?}

Traditionally, it has been generally accepted that reportability is a scientific and operational index of consciousness (Baars, 1988). A stimulus could be well accepted as having reached consciousness when participants are able to explicitly report it both in behavioral and neurological experiments (Dehaene et al., 2006). For example, in classic blindsight investigations, researchers used subjective reports to distinguish whether stimuli in different visual fields have reached awareness (Kentridge, Heywood, \& Weiskrantz, 1999, 2004). In addition, neuroscientists have also employed stimulus 
reportability as an operational definition of what is consciously perceived to study the neural correlates of consciousness (Dehaene, 2014; Sterzer, Kleinschmidt, \& Rees, 2009). However, some researchers have questioned the strong reliance on reportability to define the conscious content (Tsuchiya, Wilke, Frässle, \& Lamme, 2015). It has been argued that subjective reports necessarily require various post-perceptual cognitive functions rather than merely conscious contents, such as conceptualization, working memory storage, and response decision (Aru, Bachmann, Singer, \& Melloni, 2012; Tsuchiya et al., 2015). By adopting no-report paradigms, some researchers have isolated the neural correlates of consciousness from potential report-based confounds in neural activity (e.g., Cohen, Ortego, Kyroudis, \& Pitts, 2020; Frässle, Sommer, Jansen, Naber, \& Einhäuser, 2014). For example, using objective measurements to assess conscious perception, such as optokinetic nystagmus and pupil dilation, researchers found that frontal activation is necessary for report but not for consciousness (Frässle et al., 2014).

Consistent with the no-report paradigm, the present study provides evidence showing that participants are incapable of reporting the fully attended information that has just reached conscious awareness, suggesting that reportability might not always be appropriate to index conscious awareness. Thus, as some researchers suggested, the report-based measurements should be used in combination with no-report methods in future studies of consciousness (Tsuchiya, Frässle, Wilke, \& Lamme, 2016).

\section{Is conscious awareness sufficient for working memory?}

As both working memory and consciousness are usually indexed by accurate report, consciousness has been thought to be closely linked to working memory (Jacobs 
\& Silvanto, 2015). In fact, consciousness has been considered as a subset of working memory in several models of working memory and consciousness implicitly or explicitly (Velichkovsky, 2017). For instance, according to the activation-based working memory models (Cowan, 1999; Oberauer, 2002), working memory consists of focus of attention and activated long-term memory. Consciousness might be equated with the contents of the focus of attention, while the items in activated long-term memory could be retained unconsciously. Further, in the Global Workspace theory, consciousness has been thought as selecting internal representation within the content of working memory (Baars \& Franklin, 2003). Therefore, these theories have a common implicit assumption that a piece of consciously perceived information should be retained in working memory.

However, the results of current study, together with previous studies of attribute amnesia, suggest that conscious awareness may not be sufficient for working memory. In a previous study, we demonstrated that the report failure in the attribute amnesia studies reflects a lack of working memory consolidation of the attended information (Chen \& Wyble, 2016). This was supported by the finding that once participants had been forced to consolidate the attended attribute into working memory, the attribute amnesia effect was nearly eliminated. The current study provides further evidence that the attended information that has most likely reached a full state of conscious awareness was still not selected into working memory for report. Notably, different from previous theories assuming that conscious content could be selected into working memory through attention (Lamme, 2003), the current study suggests that sometimes even 
attended consciousness could still escape working memory.

\section{Acknowledgements}

This work was supported by grants from National Natural Science Foundation of China (No.31771201), National Science Foundation for Distinguished Young Scholars of Zhejiang Province, China (No. LR19C090002), Humanities and Social Sciences Foundation of the Ministry of Education of China (No.17YJA190001) awarded to author Hui Chen.

\section{Reference}

Aru, J., Bachmann, T., Singer, W., \& Melloni, L. (2012). Distilling the neural correlates of consciousness. Neuroscience \& Biobehavioral Reviews, 36(2), 737-746.

Baars, B. J. (1988). A cognitive theory of consciousness. Cambridge: Cambridge University Press.

Baars, B. J., \& Franklin, S. (2003). How conscious experience and working memory interact. Trends in Cognitive Sciences, 7(4), 166-172.

Block, N. (1995). On a confusion about a function of consciousness. Behavioral and Brain Sciences, 18(2), 227-247.

Block, N. (2011). Perceptual consciousness overflows cognitive access. Trends in Cognitive Sciences, 15(12), 567-575.

Brady, T. F., Konkle, T., Alvarez, G. A., \& Oliva, A. (2008). Visual long-term memory has a massive storage capacity for object details. Proceedings of the National Academy of Sciences, 105(38), 14325-14329.

Brainard, D. H. (1997). The psychophysics toolbox. Spatial Vision, 10(4), 433-436.

Chen, H., \& Wyble, B. (2015). Amnesia for object attributes: Failure to report attended information that had just reached conscious awareness. Psychological Science, 26(2), 203-210.

Chen, H., \& Wyble, B. (2016). Attribute amnesia reflects a lack of memory consolidation for 
attended information. Journal of Experimental Psychology: Human Perception and Performance, 42(2), 225-234.

Chen, H., Yan, N., Zhu, P., Wyble, B., Eitam, B., \& Shen, M. (2019). Expecting the unexpected: Violation of expectation shifts strategies toward information exploration. Journal of Experimental Psychology: Human Perception and Performance, 45(4), 513-522.

Chen, H., Yu, J., Fu, Y., Zhu, P., Li, W., Zhou, J., \& Shen, M. (2019). Does attribute amnesia occur with the presentation of complex, meaningful stimuli? The answer is, "it depends". Memory \& Cognition, 47(6), 1133-1144.

Chen, X., Hoffmann, K. P., Albright, T. D., \& Thiele, A. (2012). Effect of feature-selective attention on neuronal responses in macaque area MT. Journal of Neurophysiology, 107(5), 15301543.

Cohen, M. A., \& Dennett, D. C. (2011). Consciousness cannot be separated from function. Trends in Cognitive Sciences, 15(8), 358-364.

Cohen, M. A., Ortego, K., Kyroudis, A., \& Pitts, M. (2020). Distinguishing the neural correlates of perceptual awareness and post-perceptual processing. Journal of Neuroscience, 40(25), 4925-4935.

Cowan, N. (1999). An embedded-process model of working memory. In A. Miyake, \& P. Shah (Eds.), Models of working memory (pp. 62-101). Cambridge: Cambridge University Press.

De Brigard, F., \& Prinz, J. (2010). Attention and consciousness. Wiley Interdisciplinary Reviews: Cognitive Science, 1(1), 51-59.

Dehaene, S. (2014). Consciousness and the brain: Deciphering how the brain codes our thoughts. New York: Penguin.

Dehaene, S., \& Naccache, L. (2001). Towards a cognitive neuroscience of consciousness: basic evidence and a workspace framework. Cognition, 79(1-2), 1-37.

Dehaene, S., Bossini, S., \& Giraux, P. (1993). The mental representation of parity and number magnitude. Journal of Experimental Psychology: General, 122(3), 371-396.

Dehaene, S., Changeux, J. P., Naccache, L., Sackur, J., \& Sergent, C. (2006). Conscious, preconscious, and subliminal processing: a testable taxonomy. Trends in Cognitive Sciences, 10(5), 204-211.

Eitam, B., Shoval, R., \& Yeshurun, Y. (2015). Seeing without knowing: Task relevance dissociates 
between visual awareness and recognition. Annals of the New York Academy of Sciences, $1339(1), 125-137$.

Eitam, B., Yeshurun, Y., \& Hassan, K. (2013). Blinded by irrelevance: Pure irrelevance induced "blindness". Journal of Experimental Psychology: Human Perception and Performance, 39(3), 611-615.

Fias, W., Brysbaert, M., Geypens, F., \& d'Ydewalle, G. (1996). The importance of magnitude information in numerical processing: Evidence from the SNARC effect. Mathematical Cognition, 2(1), 95-110.

Frässle, S., Sommer, J., Jansen, A., Naber, M., \& Einhäuser, W. (2014). Binocular rivalry: frontal activity relates to introspection and action but not to perception. Journal of Neuroscience, $34(5), 1738-1747$

Jackson, J., Rich, A. N., Williams, M. A., \& Woolgar, A. (2017). Feature-selective attention in frontoparietal cortex: multivoxel codes adjust to prioritize task-relevant information. Journal of Cognitive Neuroscience, 29(2), 310-321.

Jacobs, C., \& Silvanto, J. (2015). How is working memory content consciously experienced? The 'conscious copy' model of WM introspection. Neuroscience and Biobehavioral Reviews, $55,510-519$

Kentridge, R. W., Heywood, C. A., \& Weiskrantz, L. (1999). Attention without awareness in blindsight. Proceedings of the Royal Society of London B: Biological Sciences, 266(1430), $1805-1811$

Kentridge, R. W., Heywood, C. A., \& Weiskrantz, L. (2004). Spatial attention speeds discrimination without awareness in blindsight. Neuropsychologia, 42(6), 831-835.

Kouider, S., \& Dehaene, S. (2007). Levels of processing during non-conscious perception: a critical review of visual masking. Philosophical Transactions of the Royal Society of London B: Biological Sciences, 362(1481), 857-875.

Lamme, V. A. (2003). Why visual attention and awareness are different. Trends in Cognitive Sciences, 7(1), 12-18.

Lamme, V. A. (2010). How neuroscience will change our view on consciousness. Cognitive Neuroscience, 1(3), 204-220

Li, F. F., VanRullen, R., Koch, C., \& Perona, P. (2002). Rapid natural scene categorization in the 
near absence of attention. Proceedings of the National Academy of Sciences, 99(14), 95969601.

Liu, Y., Shu, H., \& Li, P. (2007). Word naming and psycholinguistic norms: Chinese. Behavior Research Methods, 39(2), 192-198.

Marchetti, G. (2012). Against the view that consciousness and attention are fully dissociable. Frontiers in Psychology, 3, 36.

Matthews, J., Schröder, P., Kaunitz, L., van Boxtel, J. J., \& Tsuchiya, N. (2018). Conscious access in the near absence of attention: critical extensions on the dual-task paradigm. Philosophical Transactions of the Royal Society B: Biological Sciences, 373(1755), 20170352.

Oberauer, K. (2002). Access to information in working memory: exploring the focus of attention. Journal of Experimental Psychology: Learning, Memory, and Cognition, 28(3), 411-421.

Pelli, D. G. (1997). The VideoToolbox software for visual psychophysics: Transforming numbers into movies. Spatial Vision, 10(4), 437-442.

Persuh, M. (2018). Measuring perceptual consciousness. Frontiers in Psychology, 8, 2320.

Potter, M. C., Nieuwenstein, M., \& Strohminger, N. (2008). Whole report versus partial report in RSVP sentences. Journal of Memory \& Language, 58(4), 907-915.

Reddy, L., Reddy, L., \& Koch, C. (2006). Face identification in the near-absence of focal attention. Vision Research, 46(15), 2336-2343.

Reddy, L., Wilken, P., \& Koch, C. (2004). Face-gender discrimination is possible in the near-absence of attention. Journal of Vision, 4(2), 106-117.

Reynvoet, B., \& Brysbaert, M. (1999). Single-digit and two-digit Arabic numerals address the same semantic number line. Cognition, 72(2), 191-201.

Simons, D. J., \& Chabris, C. F. (1999). Gorillas in our midst: Sustained inattentional blindness for dynamic events. Perception, 28(9), 1059-1074.

Simons, D. J., \& Levin, D. T. (1998). Failure to detect changes to people during a real-world interaction. Psychonomic Bulletin \& Review, 5(4), 644-649.

Sperling, G. (1960). The information available in brief visual presentations. Psychological Monographs: General and Applied, 74(11), 1-29.

Stazicker, J. (2011). Attention, visual consciousness and indeterminacy. Mind \& Language, 26(2), 156-184. 
Sterzer, P., Kleinschmidt, A., \& Rees, G. (2009). The neural bases of multistable perception. Trends in Cognitive Sciences, 13(7), 310-318.

Tsuchiya, N., Frässle, S., Wilke, M., \& Lamme, V. (2016). No-report and report-based paradigms jointly unravel the NCC: response to Overgaard and Fazekas. Trends in Cognitive Sciences, 20(4), 242-243.

Tsuchiya, N., Wilke, M., Frässle, S., \& Lamme, V. A. (2015). No-report paradigms: extracting the true neural correlates of consciousness. Trends in Cognitive Sciences, 19(12), 757-770.

Velichkovsky, B. B. (2017). Consciousness and working memory: Current trends and research perspectives. Consciousness and Cognition, 55, 35-45.

Ward, E. J., \& Scholl, B. J. (2015). Inattentional blindness reflects limitations on perception, not memory: Evidence from repeated failures of awareness. Psychonomic Bulletin \& Review, 22(3), $722-727$.

Weiskrantz, L. (1997). Consciousness lost and found: a neuropsychological exploration. New York: Oxford University Press. 